\title{
Automatic Segmentation and Quantification of Electron Micrographs: Extracellular Components
}

\author{
Jong W. Lee, ${ }^{*}$ Edward J. Delp, $¥$ ANd Linda L. Brinkley $\dagger$ \\ ${ }^{*}$ Department of Electrical Engineering and Computer Science, and †Department of \\ Anatomy and Cell Biology, University of Michigan; and $\ddagger$ School of Electrical Engineering, \\ Purdue University
}

Received June 27, 1985

\begin{abstract}
Extracellular glycosaminoglycans when precipitated by tannic acid, appear in electron micrographs as amorphous reticulate masses or fragments sometimes finely beaded and often associated with collagen fibrils. An algorithm for automatic classification, segmentation, and quantification of the amount of tannic acid-precipitable material (TAPM) and collagen in electron microscopic images is presented. Small patches of a region are initially located and the patch boundaries are traced using a binary contour tracing algorithm. The patches are then grown out and merged together to form one large area. This area is classified using a two-dimensional feature vector into one of two classes: a region with TAPM and collagen, or one with cell bodies and/or processes. Once these areas are classificd and segmented, the distribution of TAPM is measured. The algorithm was tested on several TAPM images displaying varying amounts and configurations of TAPM with good results. It may also be adapted to process other electron microscopic images containing elements of interest which have complex or amorphous form. 1985 Academic Press, Inc.
\end{abstract}

\section{INTRODUCTION}

Extracellular matrix is thought to play a role in many developmental processes involved in morphogenesis such as cell shape changes, cell migration and displacement, and control of cell growth and differentiation [see $(I)$ and (2), for review]. The principal constituents of the extracellular matrix in embryonic tissues are the glycosaminoglycans (GAGs), mainly hyaluronate and the chondroitin sulfates. The use of tannic acid-containing initial fixatives retains the extracellular GAGs in situ. These molecules then appear in electron microscopic images as small, fine filaments associated to form reticulate masses of variable dimensions (hyaluronate), and as small, finely beaded filaments (chondroitin sulfates) (3). A method which permits the automatic identification and quantification of tannic acid-precipitable material (TAPM) would be a valuable tool for studies seeking to correlate the presence and amount of such material with local morphogenetic events.

The developing secondary palatal shelves of embryonic mice contain large 587

$0010-4809 / 85 \$ 3.00$

Copyright 1985 by Academic Press, Inc All rights of reproduction in any form reserved. 
amounts of GAGs, $60 \%$ of which is hyaluronate (4). These molecules are known to be involved in the major morphogenetic changes the shelves undergo as they remodel around the tongue to form the secondary palate (5). Thus we have used electron microscopic images of the mouse secondary palatal shelves to formulate and test our methods. An approach to automatic segmentation and classification of TAPM in such images is presented here.

There are basically two different types of areas in these electron microscopic images; those with TAPM with or without collagen fibrils, and those with cell bodies or processes. Once these areas are properly classified and segmented, the amount of TAPM in the image can be measured. The analysis is divided into two steps: (i) region classification (6), and (ii) segmentation by region growing (7). As the image is scanned small patches of cell or TAPM regions are located and merged to form one large area. A binary contour tracing technique $(8)$ is used throughout the cntire process to trace a closed boundary of an area. This method has an advantage over most other approaches, such as Fourier shape analysis (9), in that the boundary points need not be linked to form a closed boundary. Each area thus located is then classified using a set of decision rules.

One major task of all classification problems is to incorporate a priori information systematically into decision rules. Humans always use such information about an object to identify it and its background. Thus, a fundamental problem of object classification is the definition of relevant features characterizing the object. Some of the salient features of the TAPM image utilized in defining the decision rules to be employed were: cell body or process size, shape, mean gray level of the cellular components and of the extracellular space, and texture.

\section{MATERIALS AND METHODS}

Tissue preparation. The secondary palatal shelves of Day 13.5 CD-1 mouse embryos were harvested, placed in phosphate-buffered saline, $\mathrm{pH} 7.3$, cut into three segments each, and fixed for $2 \mathrm{hr}$ at room temperature in $2 \%$ glutaraldehyde in phosphate buffer containing $1 \%(\mathrm{w} / \mathrm{v})$ tannic acid to precipitate the GAGs (3) (Mallinckrodt, Kentucky). Specimens were rinsed in $0.1 \mathrm{M}$ phosphate buffer at room temperature for $1 \mathrm{hr}$, postfixed in $1 \% \mathrm{OsO}_{4}$ in $0.1 \mathrm{M}$ phosphate buffer for $1 \mathrm{hr}$, and then rinsed again in buffer. Tissues were then dehydrated in a graded ethanol series, cleared with propylene oxide, and embedded in Epon 812 (10).

Silver sections were stained with uranyl acetate for $30 \mathrm{~min}$ and lead citrate for 4-6 min and examined and photographed using a Philips 400 electron microscope.

Image acquisition and characteristics. The photographic negatives of the EM images were digitized using an Optronics rotating drum, flying spot scanner, with a spot size of $50 \mu \mathrm{m}$. The images were recorded as a matrix of brightness values of $512 \times 482$ picture elements (pixels) in size. The final magnification of the digitized images was 50,000 . 


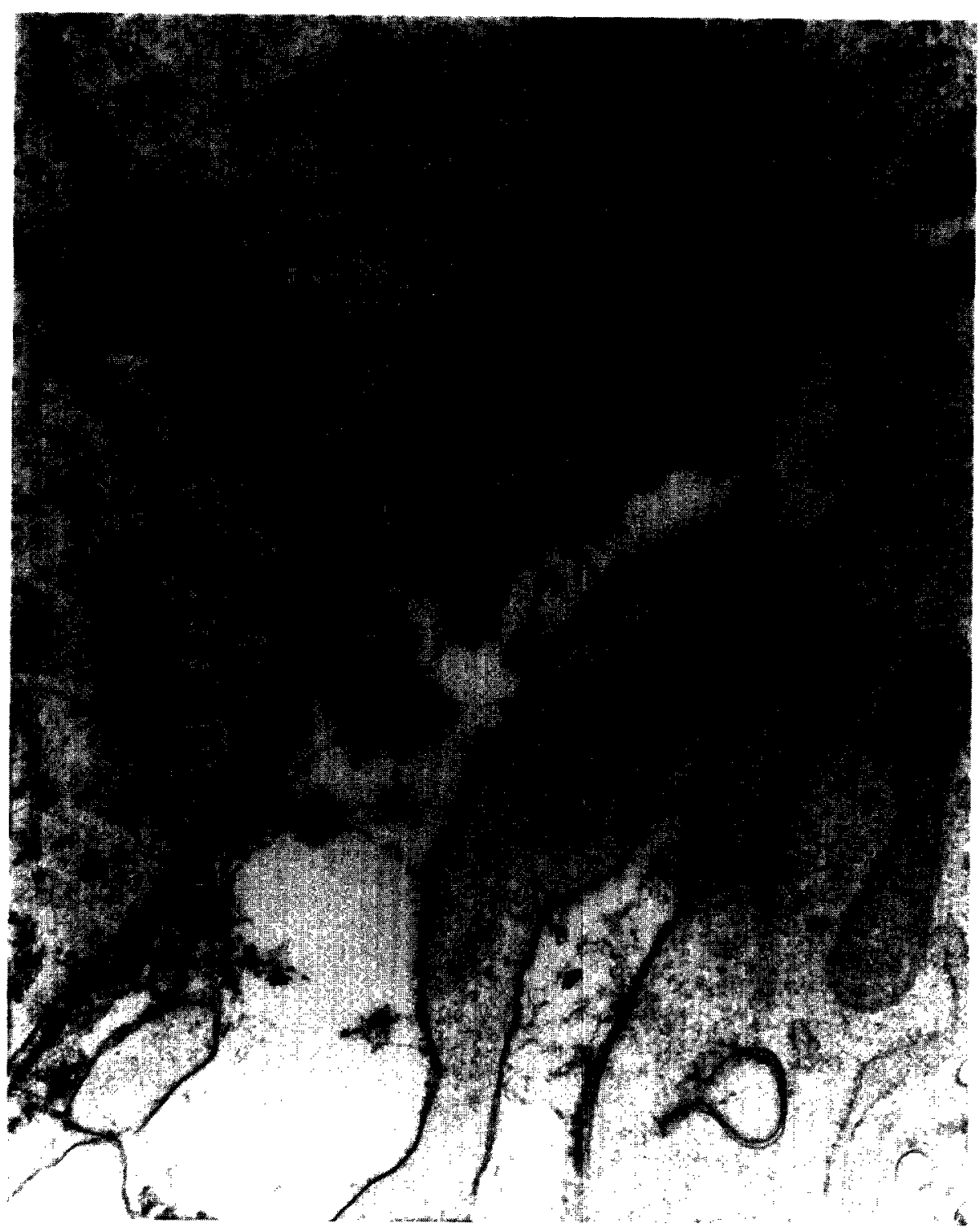

FIG. 1. An original EM image at $29,500 \times$ magnification. E, epithelium; M, mesenchyme; *, lamina densa; $\$$, TAPM; $\leftarrow$, collagen.

A typical image used to develop the algorithm is shown in Fig. 1. The image consists of epithelium with its underlying basal lamina, mesenchymal cells and their processes, and extracellular space containing TAPM and collagen in various planes of section. Segmentation of the image prior to measurement of the TAPM requires that the cells be differentiated from extracellular space (background). Next the two main features of the background, collagen and TAPM, 
must be discriminated from it as well as from one another. The salient features of the cells are (i) edges of fairly uniform thickness, (ii) edge elements of relatively low gray values, (iii) high variance or busyness of gray values inside the cell, and (iv) usually round or elliptical shapes. The prominent features of the background area are (i) high mean gray value, (ii) low variance in gray values across the region, and (iii) irregular boundary shapes. Collagen appears as long strands or discrete small, round shapes of low gray value while TAPM appears as a fuzz-like substance. The variable appearance of collagen molecules is due to their random orientation in three-dimensional space.

Definitions. Some commonly used features ( 11 ) for biological image classification were considered for the EM images. Those features that yielded the least classification error, based on experimental results, were chosen as the features used in the analysis. In contrast to most biological images analyzed such as blood cells, the number of features for the EM images are limited as filtered color images cannot be obtained with the electron microscope. Three types of features were defined and grouped as follows: (i) density features; (ii) geometric features, and (iii) texture features. In the forthcoming discussion, $g(i, j)$ is the image gray value of an image at point or pixel $(i, j), S_{c}$ is the cellular region, $S_{b}$ is the background area, $P_{c}$ is the cell boundary, and $P_{b}$ is the background boundary. The cell and background boundaries are traced using the binary contour tracing method discussed later. The boundary length and the inside area are measured by counting the number of pixels.

Density features.

Average density of cell:

$$
d_{c}=\frac{1}{a_{c}} \sum_{(i, j) \in S_{i}} g(i, j) .
$$

Average density of background:

$$
d_{b}=\frac{1}{a_{b}} \sum_{(i, j) \in S_{b}} g(i, j) .
$$

Standard deviation of cell:

$$
\sigma_{c}=\left[\frac{1}{a_{c}} \sum_{(i, j) \epsilon S_{c}}\left[g(i, j)-d_{c}\right]^{2}\right]^{1 / 2} .
$$

Standard deviation of background:

$$
\sigma_{b}=\left[\frac{1}{a_{b}} \sum_{(i, j) \epsilon S_{b}}\left[g(i, j)-d_{b}\right]^{2}\right]^{1 / 2} .
$$

Geometric features.

The cellular area:

$$
a_{c}=\sum_{(i, j) \in S_{c}} 1
$$

The background area: 


$$
a_{b}=\sum_{(i, j) \in S_{b}} 1
$$

The cell perimeter:

$$
p_{c}=\sum_{(i, j) \in P_{c}} 1
$$

The background perimeter:

$$
p_{b}=\sum_{(i, j) \in P_{b}} 1 \text {. }
$$

Texture features. If in a $3 \times 3$ neighborhood in an image matrix, gray level values are represented as below,

$$
\left[\begin{array}{lll}
A & B & C \\
D & E & F \\
G & H & I
\end{array}\right] .
$$

The busyness $(10)$ can defined by $\min \left(v_{x}, v_{y}\right)$ as follows:

$$
\begin{aligned}
& v_{x}=|A-B|+|B-C|+|D-E|+|E-F|+|G-H|+|H-I| \\
& v_{y}=|A-D|+|D-G|+|B-E|+|E-H|+|C-F|+|F-I|
\end{aligned}
$$

where $\min \left(v_{x}, v_{y}\right)$ is the minimum of $v_{x}$ or $v_{y}$. The busyness is a measure of the number of transitions from a uniform region to another with different properties. If the property of interest is the gray value, then the number of transitions from a black pixel to a white one, or white to black, will yield the degree of busyness of an image. A region with salt and pepper type of noise (12) is very busy whereas a region with uniform gray value is not. The variance of the gray values of a region also yields some measure of busyness, but one which is not as definitive as the transitional measure. For instance, a region with two sections of bright and dark gray values and a region with four sections of bright and dark will yield the same variance (thus the same degree of busyness) whereas the transitional feature will clearly show that the latter case is busier. For simplicity the absolute difference gradient operator may be substituted for the busyness,

$$
v_{x y}=|\boldsymbol{B}-\boldsymbol{E}|+|\boldsymbol{D}-\boldsymbol{E}| .
$$

The components of the feature vector. Typically, the features are combined in a feature vector $(11)$. This feature vector is used to determine which regions of the image belong to TAPM, cell bodies or processes, or the background space. Based on cxperimental results, it was determined that only three of the features discussed above were needed. These features were mean gray value, perimeter, and busyness. The perimeter was used to condition the busyness of a region so as to yield better classification results. The final feature vector has two components. A two-dimensional feature vector 


$$
\vec{F}=\left[\begin{array}{l}
f_{1} \\
f_{2}
\end{array}\right]
$$

is defined from the features described above where:

$$
\begin{aligned}
& f_{1}=d_{i} \\
& f_{2}=\hat{f}_{2} \cdot w
\end{aligned}
$$

and where

$$
\hat{f}_{2}=v_{x y_{i}} \quad i \in(\text { cell, background })
$$

and

$$
w=\left\{\begin{array}{cl}
C_{1} / p_{i} & \text { if } p_{i}<C_{0} \\
1 & \text { otherwise }
\end{array}\right.
$$

where $C_{0}$ and $C_{1}$ are constants determined experimentally to yield the best classification results, and $p_{i}$ is the perimeter of a cellular region or background space containing TAPM. The first feature $f_{1}$ is the mean gray value of a region. The second feature is the busyness of a region. This feature is obtained first by measuring the busyness, $v_{x y}$, of a region as discussed earlier, and then is conditioned by the perimeter of the region under test. This is reasonable since the small cellular regions tend to be smoother than the larger ones, so that some normalization is necessary. The region can now be classified using a decision rule such as the Bayes or the maximum likelihood classifier (11). The decision rule is

$$
\left(\vec{F}-\vec{M}_{c}\right)^{T} \cdot \Sigma_{c}^{-1} \cdot\left(\vec{F}-\vec{M}_{c}\right)+C_{4}<\left(\vec{F}-\vec{M}_{b}\right)^{T} \cdot \Sigma_{b}^{-1} \cdot\left(\vec{F}-\vec{M}_{b}\right)
$$

where $\vec{F}$ is the feature vector of a region, $\vec{M}_{i}$, and $\Sigma_{i}^{-1}$ is the mean and the inverse covariance matrix for the cell or the background, and $C_{4}$ is an empirical constant. The above equation represents a general form of the minimum distance classifier $(10,11,13)$. The values of the two features, i.e., the feature vector, are compared against typical values of the two EM image regions, the cell and the background. The region which yields the least difference is chosen as the correct class of region. Thus, if Eq. [17] is satisfied, then the region under test is classified as a cell; otherwise, as background. Before this type of classification method described by Eq. [17] can be used, one must know the values of $d_{b}, d_{c}, \sigma_{b}$, and $\sigma_{c}$. These are obtained using many test samples, where the correct classification of each $\vec{F}$ is known a priori. This data is known as training data and must be reliably obtained (II).

Contour tracing. Before a region can be classified as being part of a cell or the background, potential regions must be found in the image. This process is known as segmentation (11). Equations [12]-[18] are computed and the regions are classified using Eq. [17]. Once the potential regions are segmented using region growing (11), the boundary points are obtained using contour tracing. The region is grown out by tracing the boundary by successively decrementing 
the threshold in our contour tracing algorithm. If an image, after the threshold operation at some value, $t$, in a $3 \times 3$ neighborhood is represented by a chain code $(8)$ as shown below, the boundary can be traced as follows:

$$
\begin{aligned}
& 670 \\
& 5 \times 1 \\
& 432
\end{aligned}
$$

If the center pixel marked " $x$ " is the current boundary point, and the previous boundary point is 7 , the next point is found by scanning the neighborhood pixels clockwise starting from a pixel location that is $90^{\circ}$ to the left of the direction from which the current pixel arrived. The starting pixel here is 1 . As the neighboring pixels are scanned, the first pixel exceeding a threshold value is chosen as the next boundary point. An example is shown below for clarity. Assuming that a region under test has been thresholded to yield a bimodal image shown below, the boundary of the area marked as 1 is traced as follows:

$$
\begin{array}{lllllll}
a & b & c & d & e & f \\
0 & 0 & 0 & 0 & 0 & 0 & h \\
0 & 1 & 0 & 0 & 0 & 0 & i \\
0 & 1 & 1 & 1 & 1 & 0 & j \\
0 & 1 & 1 & 1 & 1 & 0 & k \\
0 & 0 & 0 & 0 & 0 & 0 & 1
\end{array}
$$

Assuming the current boundary point is at $(\mathrm{b}, \mathrm{i})$, and the previous boundary point was $(b, j)$, the next point is chosen by scanning the image clockwise from the point $(\mathrm{a}, \mathrm{i})$. The direction from the previous point to the current is up and the point that is $90^{\circ}$ to the left of the direction is $(\mathrm{a}, \mathrm{i})$. The points $(\mathrm{a}, \mathrm{i}),(\mathrm{a}, \mathrm{h}),(\mathrm{b}, \mathrm{h})$, $(\mathrm{c}, \mathrm{h})$, and $(\mathrm{c}, \mathrm{i})$ are first tested to see if its value is 1 . Since they are not, the next point $(c, j)$ is tested in sequence. Since it is 1 , the point $(c, j)$ is chosen as the next boundary pixel. Now the direction from the old point, $(b, i)$, to the new point, $(\mathrm{c}, \mathrm{j})$, is $-45^{\circ}$. The starting point for the next houndary pixel search is again found by locating the point that is $90^{\circ}$ to the left of this direction, and it is found to be $(\mathrm{d}, \mathrm{i})$. By repeating the process, the boundary of a region can be successfully traced. The process is terminated if the next boundary point coincide with the first starting point.

\section{The Algorithm}

In this section we describe in detail the algorithm used in our studies. A small patch of a region is first located and its boundary is determined. The feature vector of the area is then computed to classify the region properly. Once it is classified, its neighboring pixels are merged by region growing to form one large area. To increase certainty and efficiency, the algorithm is split up as a hierarchial two-pass process. 
Preprocessing. Since the gray levels in the EM images vary from image to image, it is necessary that the gray levels be normalized for consistency. Various approaches, such as simple stretching and histogram equalization were examined. Simple histogram stretching of the image along with a slight compression of gray levels at very high and low gray values yielded an acceptable result. By compressing the low gray values, the boundaries of cellular processes were accentuated. Compressing the high gray values resulted in better overall normalization since the number of pixels at high gray values was small. The gray values which were two standard deviations away from the mean value were found to be optimal. Therefore, the pixel gray values above and below these gray values, were set to 255 (white) and 0 (black), respectively. Let $h(i, j)$ represent the image after it has undergone transformation and let $g(i, j)$ be the original image. The image $h(i, j)$ is computed as follows:

$$
h(i, j)= \begin{cases}\left(\frac{G_{\mathrm{EM}}}{2 \sigma_{\mathrm{EM}}}\right)\left[g(i, j)-G_{\mathrm{lo}}\right] & \text { if } g(i, j)<G_{\mathrm{EM}} \\ \left(\frac{255-G_{\mathrm{EM}}}{2 \sigma_{\mathrm{EM}}}\right)\left[g(i, j)-G_{\mathrm{hi}}\right]+G_{\mathrm{EM}} & \text { if } g(i, j)>G_{\mathrm{EM}}\end{cases}
$$

with

$$
\begin{aligned}
& G_{\mathrm{lo}}=G_{\mathrm{EM}}-2 \sigma_{\mathrm{EM}} \\
& G_{\mathrm{hi}}=G_{\mathrm{EM}}+2 \sigma_{\mathrm{EM}}
\end{aligned}
$$

where $\sigma_{\mathrm{EM}}$ and $G_{\mathrm{EM}}$ are the standard deviation and the mean of $g(i, j)$, respectively. This results in a nonlinear stretching of the image with the lower and upper gray levels of the image being slightly compressed.

First pass: The background segmentation. The main purpose of this step was to accurately locate and classify the background areas. This is important for the information obtained was used as a reference to check the closure of a region.

If the image in Fig. 1 is initially thresholded such that all the pixels exceeding a threshold value are mapped into one and the others into zero, a bimodal image shown in Fig. 2 is obtained. Since the background areas have high mean gray level and low variance, they appear as chunks of closed, smooth white region. The cells do not behave as nicely because the gray values inside the cells are highly irregular; however, the edge elements are almost always darker than the rest of the cells so that the cells also appear as closed areas with somewhat irregular shapes. Since each region has different mean gray values, even among the same type of areas, a high initial threshold level is chosen such that almost all background regions are clearly separated from the cellular regions as shown in Fig. 2. If the threshold level was too low, then some of the cellular and background areas may appear as a single blob as shown in Fig. 3 . Notice that some cells that appeared as a black patch in Fig. 2 now show up as closed regions with white inside. Since the images analyzed are normalized so that the gray levels are consistent for all images, an optimal threshold level can be found which works for all images. 


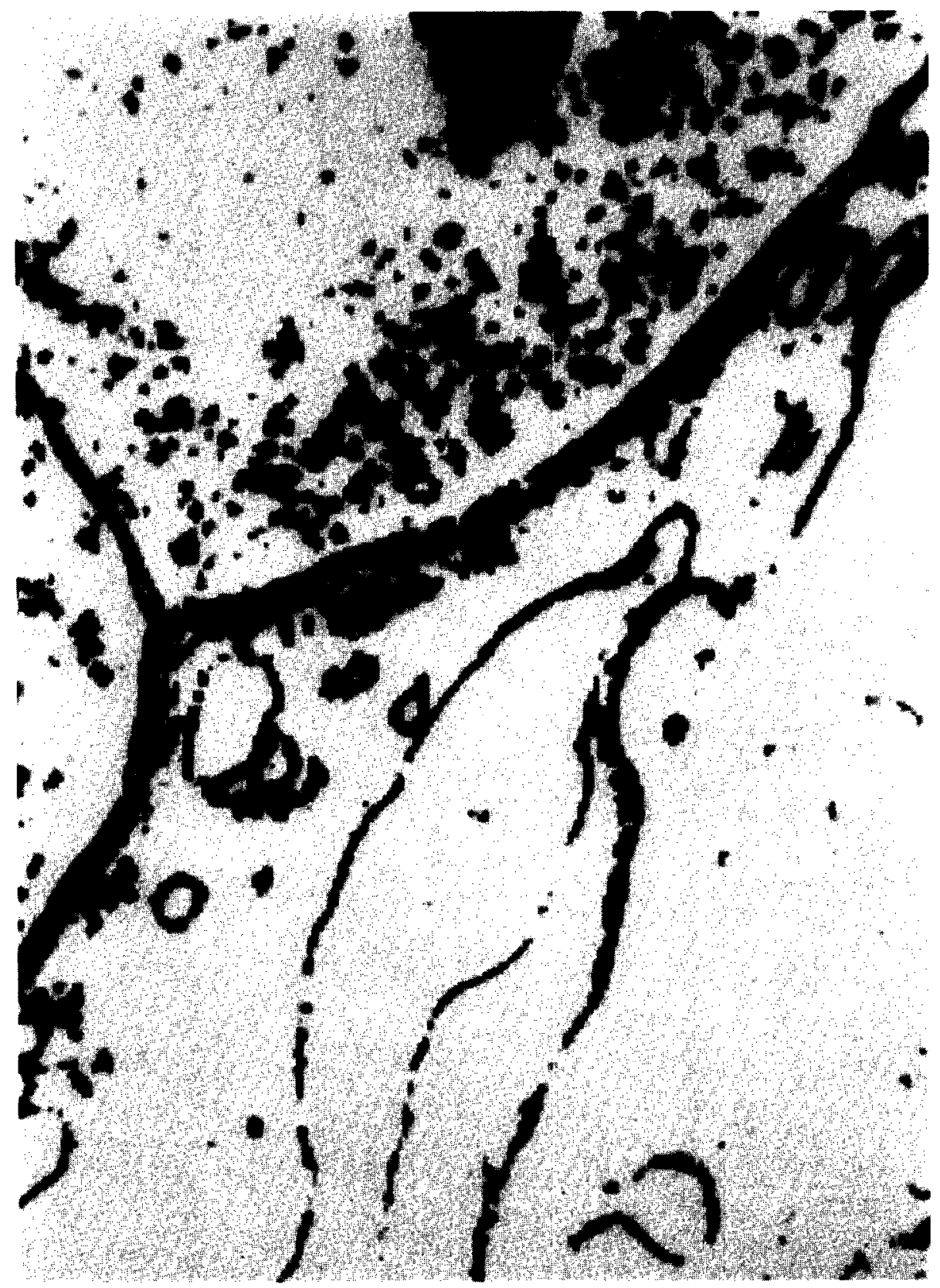

Fir. 2. Image in Fig. 1 thresholded at $t=200$.

Once these areas are located and the boundaries traced using the contour tracing algorithm, the statistics of the enclosed areas are computed and the areas are classified into one of the two classes: cell and cell processes or 


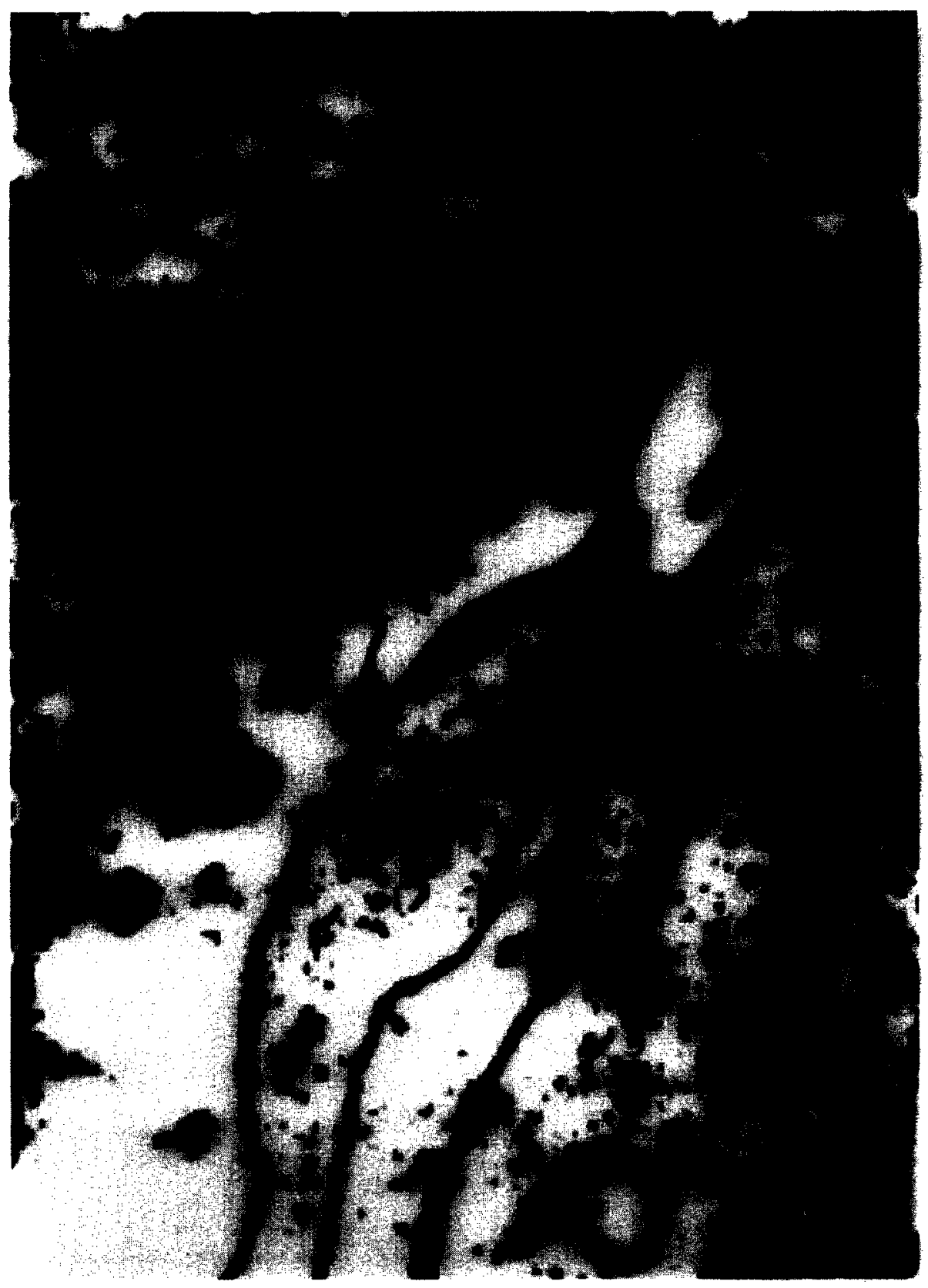

FIG. 3. Image in Fig. 1 thresholded at a too-low value.

background. This information is then mapped into a look-up matrix of the same size as the original image to be used in the second pass of the algorithm. This process is explained as follows: 
(1) Using a window of $N \times N$ size, the image is scanned from top to bottom with an initial threshold value $t_{k}$.

(2) A small patch is located such that

$$
\begin{aligned}
& W=\text { image region in the window } \\
& g(i, j)>t_{k} \quad(i, j) \in W
\end{aligned}
$$

(3) The area which encloses the patch is traced with the threshold value $t_{k}$.

(4) This area is checked for closure. If it is not closed, this patch is rejected and the image is scanned for a new patch. If it is closed, the statistics of the enclosed area are computed for classification. This information is entered in the look-up matrix for later use.

(5) This sequence is repeated on succeeding patches.

The window size $N$ can be chosen such that it only locates the white patches exceeding a certain minimum area. This helps eliminate very small patches, which may be abundant in a noisy image.

It is not important that the shape of the background areas traced are exact; that is only the major portion of the background areas need be identified at this stage. Because a high threshold level was chosen, only those cells with high inside gray level are located initially. They are only located for program efficiency so that no time is wasted identifying these cells again, as the rest of the cell will be picked up later in the second pass.

Second pass: Segmentation by region growing. Because of the irregularities within the cells, the cellular areas traced in the first pass appear as patches of irregular shapes. The boundaries obtained so far only contain portions of the inside of the cells. These areas need to be expanded by region growing as mentioned earlier, to obtain smooth edge boundaries surrounding the whole cell. This process is explained as follows:

(1) The first three steps in pass one are repeated.

(2) This area is checked for closure. If it is not closed, this patch is rejected and the image is scanned for a new patch. If it is closed, then the threshold level $t_{k}$ is lowered and the boundary is traced again with this new threshold value. The process is repeated as long as the boundary remains closed. If the threshold value is lowered too much, the edge boundary breaks up. When this occurs, the threshold level is increased back to the previous level and the contour retraced. This information is entered in the look-up matrix.

(3) These steps are repeated until the entire image has been processed.

After the entire image is processed, only the cells that have patches with their gray values higher than the initial threshold level, $t_{k}$, and areas bigger than the scanning window are located and classified. To check the remaining cells, the initial threshold level is decremented by a fixed amount, and the image is scanned again, to pick up the cells with lower gray values. Experimental results indicate that about three iterations are required to identify all the cell processes 

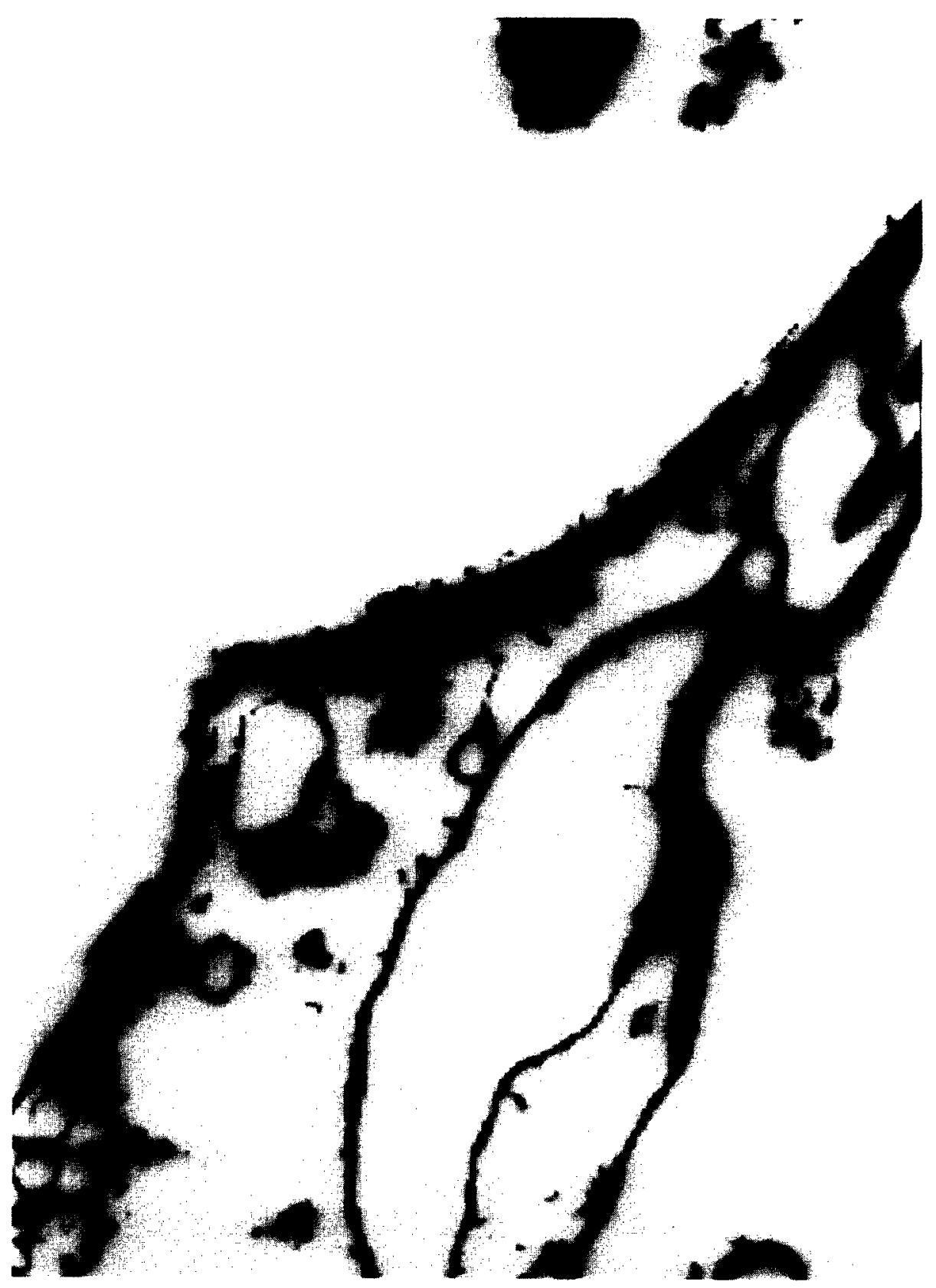

FIG. 4. Look-up matrix image.

in these images. Figure 4 shows the look-up matrix for the image after all cell processes have been identified.

At this point the traced cell boundaries only contain the areas within the cell 

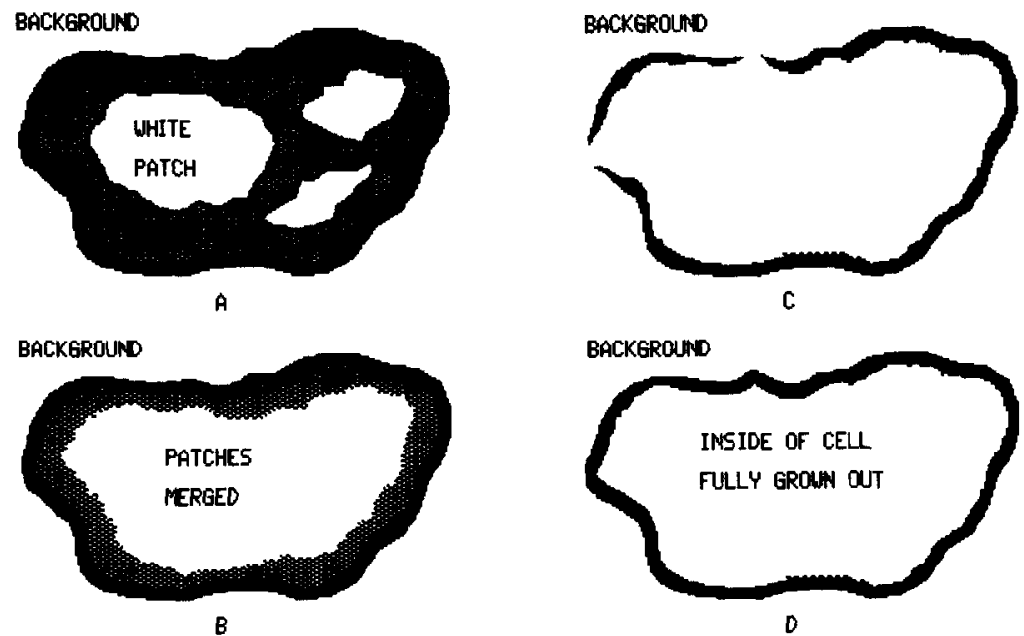

Fin. 5. (A) A cell with inside patch traced with an initial threshold level $t_{i}$. (B) $t_{i}$ decremented and boundary retraced. (C) $t_{i}$ decremented too much. The edge breaks up and all four directions about a pixel being traced is checked for open edge. If in any direction, a patch marked as background is encountered, the edge is open. (D) Final boundary traced.

and exclude the edge pixels. For a large cell, the gray values for the edge pixels fluctuate from one side of the cell to the other, and the fluctuation may be severe in some cases. Since a single threshold value is used to contour trace the boundary at one time, the inside patch may not be fully grown out to the true edge contour in some spots. This problem is overcome by sliding a window of a varying size along the already traced boundary contour, and by applying the same technique mentioned above within the window. Since it is reasonable to assume that the pixel gray levels are highly correlated within a small neighborhood, a minimum threshold value can be found within the box which will push the boundary contour out to the true edge contour.

To complete the segmentation process, all the information obtained thus far is entered in the look-up matrix, and the entire cell bodies are now stored in the matrix. The original image is compared against the look-up matrix and the TAPM regions are segmented out to produce the final image. These steps are illustrated in Figs. 5A through $\mathrm{C}$ and the look-up matrix and the final segmented image is shown in Figs. 6 and 7, respectively.

Separation and measurement of TAPM. Collagen fibrils and TAPM are first separated from one another. Collagen fibrils have very low gray values and occupy 0-20 pixel values; they are removed by thresholding the image accordingly. Background regions now contain only the TAPM itself. The histogram of such regions have a pronounced valley. The TAPM is measured by thresholding the image at a level which is determined by locating the minimum dip of the gray level histogram for that region. This technique is used in many applications to classify different regions of an image such as blood cells (13). The area 


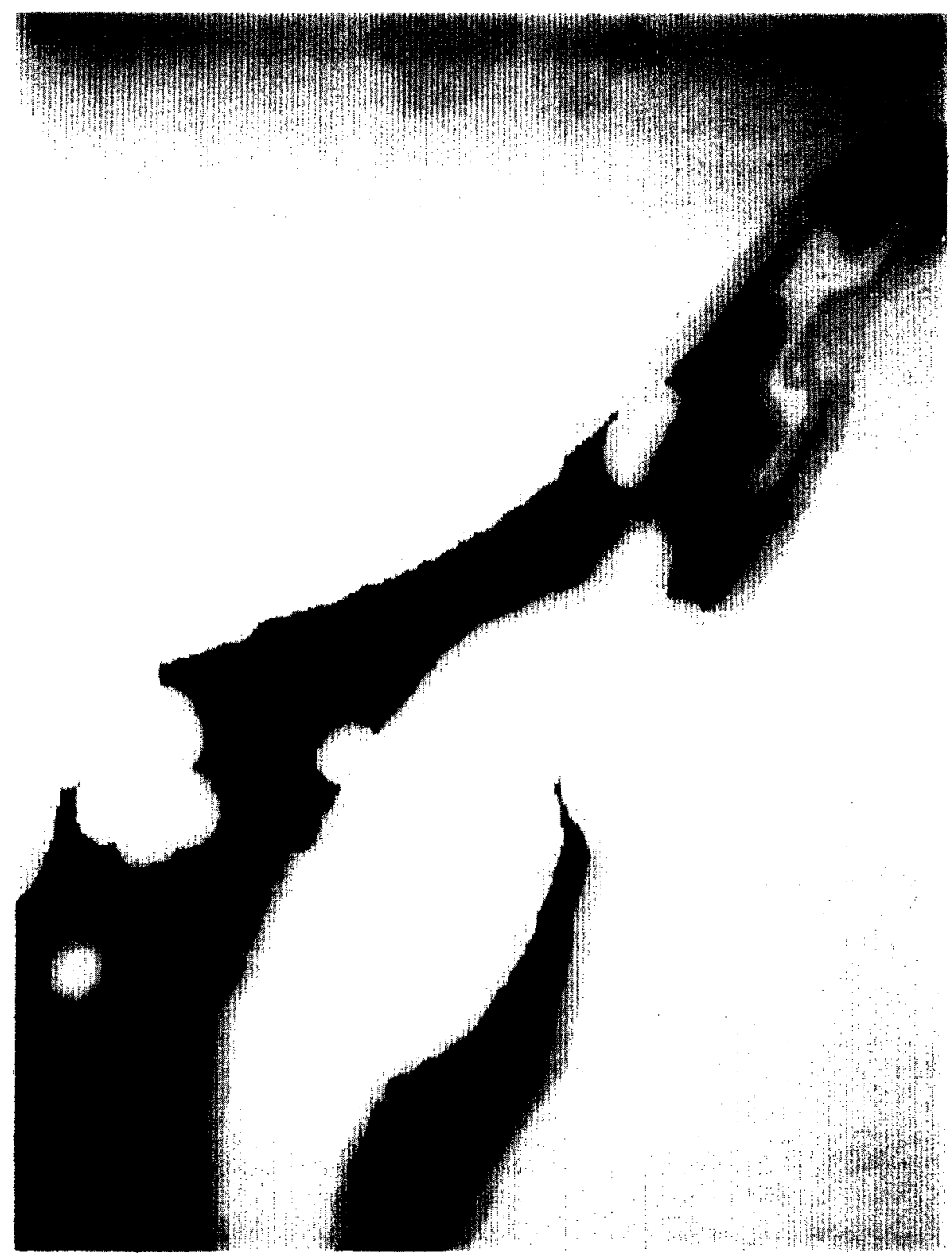

FIG. 6. The final look-up matrix.

of the image covered by the background, collagen and TAPM are then computed and the results reported as percent of total background area covered by TAPM or collagen. 


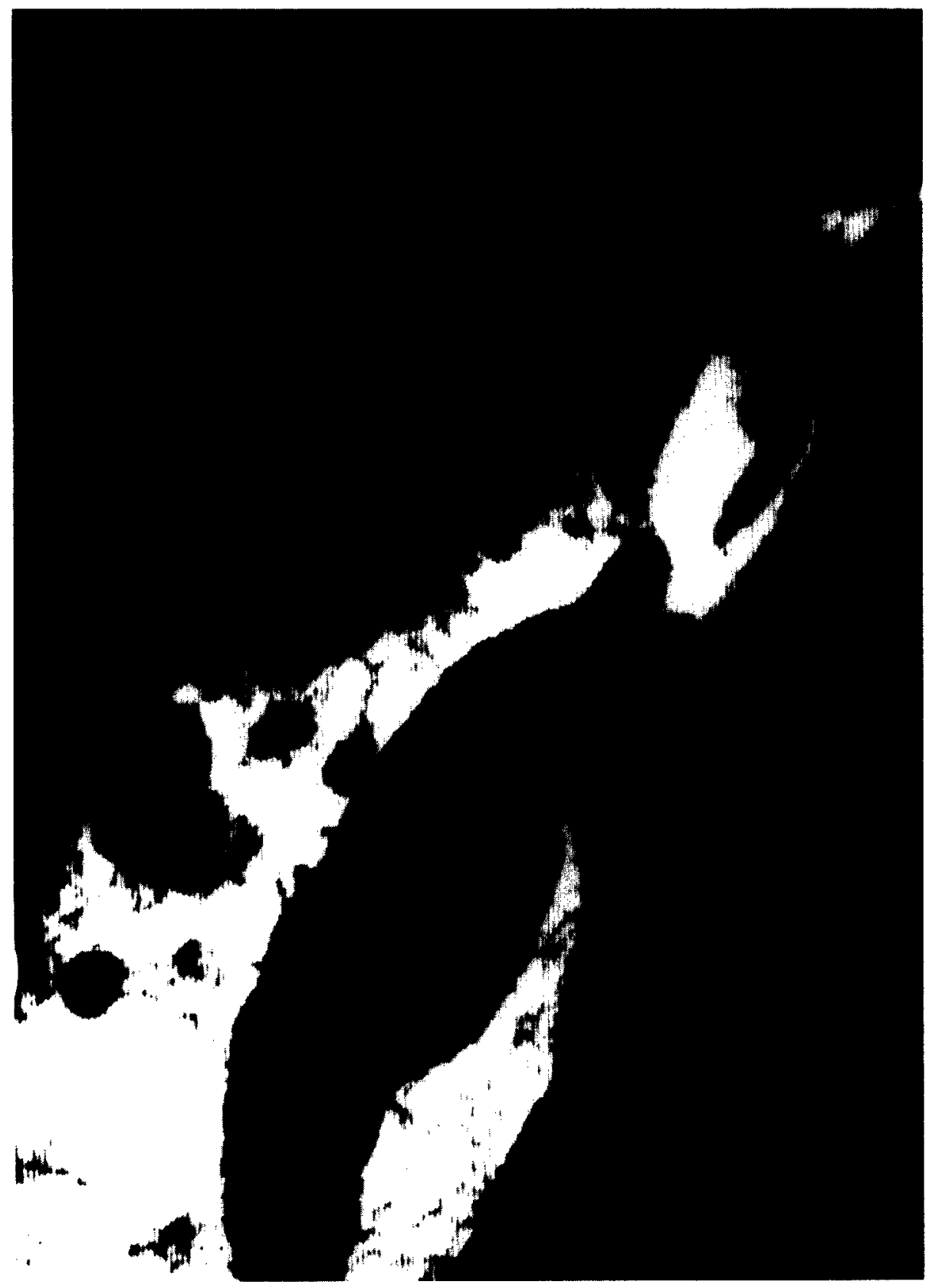

FIG. 7. The final segmented EM image. 


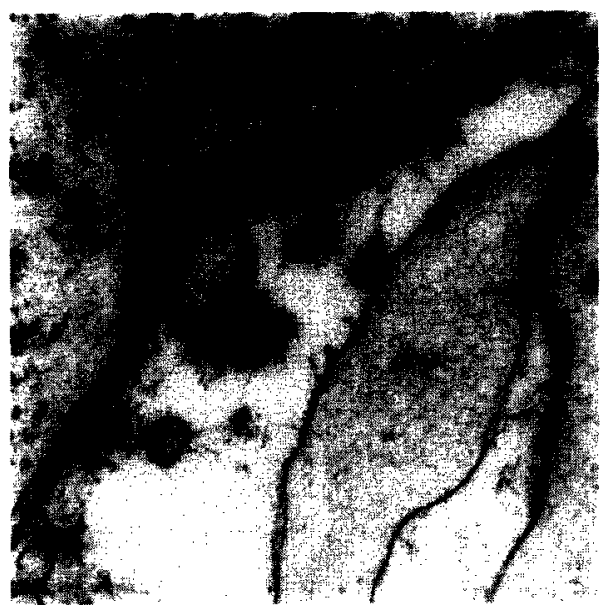

$17.8 \%$ TAPM, $2.3 \%$ Collagen

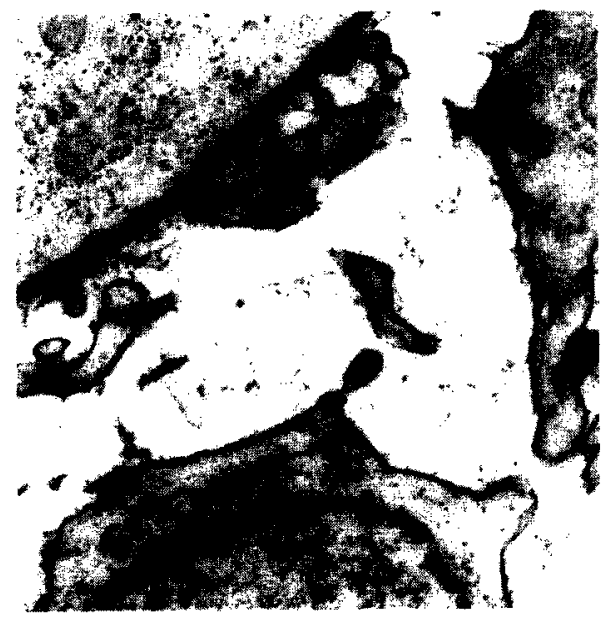

6.3\% TAPM, 3.1\% Collagen

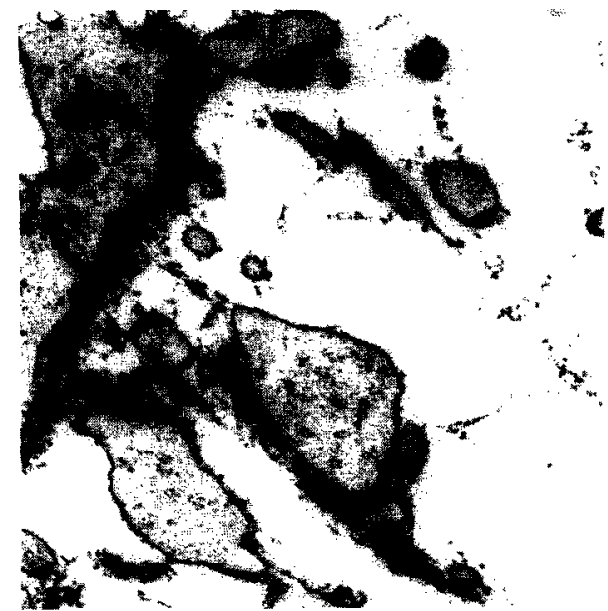

7\% TAPM. $2.3 \%$ Collagen

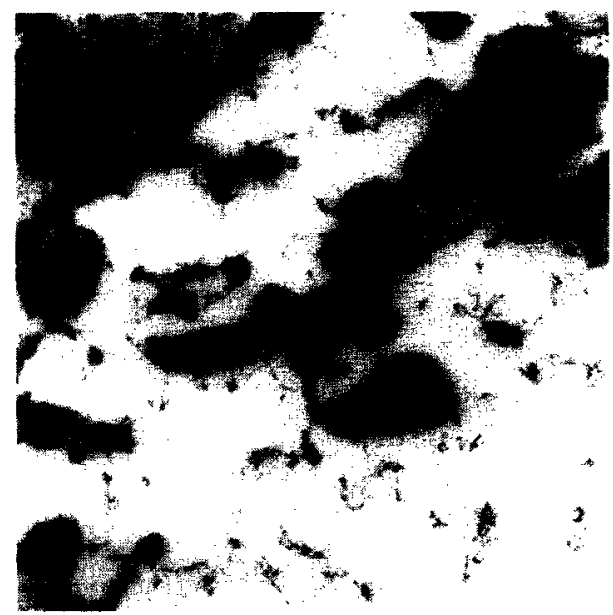

$4.4 \%$ TAPM, $2.3 \%$ Collagen

FIG. 8. Four electron micrographs analyzed by the algorithm. The amount of tannic acid-precipitable material (TAPM) and collagen is expressed as percentage of extracellular space covered.

\section{RESUltS}

The algorithm was tested on six EM images. The average time to process each digitized image is $10 \mathrm{man}$ min, with an average VAX 11/780 CPU time of $0.6 \mathrm{sec}$. Four of the images and the amount of TAPM and collagen determined to be in each are shown in Fig. 8. We compared the algorithm's findings with hand measurements of the substances of interest. To do this, the background areas of the images, the collagen fibrils and TAPM were traced on a bit pad linked to a Tektronix 4054 running a simple micrograph measurement program. The percentages of extracellular space occupied by TAPM and collagen were 
then calculated. This manual method was selected as it is the closest equivalent to what the algorithm itself does.

Even when the micrographs were enlarged many times that of the negatives digitized for the algorithm, measurement done by hand consistently overestimated the amount of TAPM by factors ranging from 1 to 3 , while giving an approximately eight-fold underestimation of collagen. The features were simply too small and/or amorphous to be reliably traced by hand. The average time to complete manual measurements and calculations was $30 \mathrm{~min}$ per photograph, not including the time required to print the photographs.

\section{Discussion}

The algorithm provides a simple, rapid, and reliable mean of automatically segmenting cellular from extracellular components, and identifying and quantifying TAPM and collagen in digitized images of electron micrographs. This permits large numbers of such micrographs to be analyzed in a timely fashion for any given experiment, something that was previously not possiblc. This provides a useful technique for studies which seek to correlate the amount of TAPM with morphogenetic change in a particular region, or to judge the local effects of experimental manipulations of these molecules. The method is also adaptable to the identification and quantification of any components of photographic images which can appear as isolated or complex collections of strands and particulate components.

\section{ACKNOWLEDGMENTS}

This research was partially sponsored by NIH Grants DE02774 and 5K04DE00104 to L.L.B. and by the Biomedical Research Council and the Office of the Vice-President for Research, University of Michigan.

\section{REFERENCES}

1. HAY, E. D. (Ed.) "Cell Biology of Extracellular Matrix," p. 417. Plenum, New York, 1981.

2. Trelstad, R. L. (Ed.) "The Role of Extracellular Matrix in Development," p. 643. Liss, New York, 1984.

3. Singley, C. T., AND Solursh, M. The use of tannic acid for the ultrastructural visualization of hyaluronic acid. Histochemistry 65, 1493 (1980).

4. Pratt, R. M., Goggins, J. R., Wilk, A. L., anv King, C. T. G. Acid mucopolysaccharide synthesis in the secondary palate of the developing rat at the time of elevation and fusion. Dev. Biol. 32, 230 (1973).

5. Brinkley, L., ANd Morris-Wiman, J. The role of extracellular matrices in palatal shelf closure. In "Current Topics in Developmental Biology" (E. F. Zimmerman, Ed.), Vol. 19, pp. 17-36. Academic Press, New York, 1984.

6. Blanz, W. E., And Reinharnt, F. R. Image segmentation by pixel classification. Pattern Recogn. 13, 293 (1981).

7. GuPTA, J. N., AND Wintz, P. A. A boundary finding algorithm and its applications. IEEE Trans. Circuits Syst. CAS22, 351 (1975).

8. Cahn, R. L., Poulsen, R. S., And Toussaint, G. Segmentation of cervical cell images. Histochem. Cytochem. 25, 681 (1977). 
9. Rosenfeld, A.. And Kak. A. C. "Digital Picture Processing." 2nd ed.. Vol. 2. Academic Press, New York, 1982.

10. LuFT, J. Improvements in epoxy resin embedding methods. J. Biophysic'. Biochem. ('vrol. 9. 409 (1961).

11. MUI. J. K., AND FU, K. S. Automated classification of nucleated blood cells using a binary tree classifier. IEEE Trans PAMI 2, 429 (1980).

12. Huang, T. S. "Two Dimensional Digital Signal Processing II," Vol. 43. Springer-Verlag. Berlin/Heidelberg, 1981.

13. Prewitt, J. M. S., and Mendelsohn, M. L. The analysis of cell images. Trans. N.Y. Accad. Sri. 128, 1075 (1966) 